\title{
REPORT OF THE WORKING GROUP ON NUMERICAL DATA
}

1. Origin of the Group. The IAU Executive Committee set up a small Working Group, under the chairmanship of Dr. Charlotte Moore-Sitterly, to consider the problems of collecting and disseminating numerical data in astronomy and astrophysics. This Group organised two open meetings at the IAU General Assembly at Brighton in August 1970 and as a result of the widespread interest shown in this subject it was decided that a permanent Group should be set up, with Dr. G. A. Wilkins as its first chairman. The report of the preliminary Group is published in Trans. IAU, 14B (1970), 245-8, 1971; it contains a list of the data centres that were reported to the Group at the time.

The formation of the permanent Group was delayed until the Chairman had discussed its constitution with the President of the Union and his proposals had been discussed and amended by the Executive Committee. The agreed statement on the organisation and activities of the Group is as follows:

(a) The activities and membership of the Working Group shall be determined by the Executive Committee of the Union.

(b) The principal aims of the Group shall be:

(i) to collect and publish information about the existence and preparation of files of numerical data, especially those in machine-readable form, that are relevant to the interests of the Union; and

(ii) to recommend ways by which the preparation of such data files and the retrieval of information from them may be carried out economically and effectively, and to offer advice on these matters to the appropriate Commissions.

In carrying out these activities the Group shall endeavour to complement, and not duplicate, the work of the Commissions of the Union in their specialised fields.

(c) The Group shall consist of a Chairman and not more than twelve members of the Union, one of whom shall be appointed Deputy Chairman. The Chairman shall co-ordinate the activities of the members of the Group and shall report to the Executive Committee of the Union before each General Assembly. Each member of the Group will normally be responsible for reviewing relevant activities in one or more of the fields of interest of the Group in addition to taking part in the general discussions within the Group.

The Chairman and members of the Group shall retire at the beginning of each General Assembly of the Union, but may be re-appointed.

(d) The Group shall normally meet at each General Assembly to decide the basis of its activities during the following three-year period. The Group shall normally work by correspondence between General Assemblies, but the Chairman may convene a special meeting if necessary.

(e) The Group may maintain a list of consultants (not necessarily members of the Union) who are willing to provide information and advice on particular topics.

(f) Expenses incurred by members of the Group in connection with its activities will only be met by the Union if prior approval has been obtained from the Executive Committee on the recommendation of the Chairman of the Group.

The number of members of the Group has been deliberately restricted so that regular correspondence and round-table discussions are feasible. The members are to be well distributed by both field of interest and country of origin. The members are not nominated representatives of particular Commissions, but will endeavour to maintain a two-way flow of information between all interested Commissions and the Group. It is realized that twelve astronomers cannot be expected to provide expert coverage of all the relevant fields of astronomy and physics, but the panel of consultants provides information and advice to the Group through individual members or the Chairman. Other astronomers are also invited to send information or comments that they consider would be useful for example, to report the existence of special data files or techniques. It is also to be expected that 
the Group will organise open meetings at each General Assembly for the discussion of the reports of the Group and for the presentation of invited papers on topics of general interest.

2. Membership of the Group. The following list gives the names of those who have agreed to be members of the Group and the fields for which they have provisionally accepted responsibility for compiling information about data activities.

W. A. Baum
M. S. Davis
D. S. Evans
R. H. Garstang
C. O. R. Jaschek
J. Jung
K. I. Kellerman
T. Lederle
B. Morando
J. B. Sykes
Z. Kadla (Mrs.)
G. A. Wilkins

physical data on planets

celestial mechanics; information on retrieval

general topics

fundamental spectroscopic data; solar physics

stellar spectra and photometry

stellar data

radio astronomy; X-ray astronomy, etc.

stellar positions, parallaxes and motions

solar system position data

documentation

data files, etc., in U.S.S.R. and Eastern Europe

general matters and topics not otherwise covered by above.

The following persons have agreed to act as consultants; or have otherwise supplied information and advice:
A. N. Argue
P. B. Fellgett
J. D. Mulholland
A. H. Batten
H. Gursky
G. H. Pettengill
W. P. Bidelman
B. Hauck
J.-P. Rozelot
R. Cayrel
K. Haramundanis (Mrs.)
J. W. Chamberlain
J. A. Hynek
L. Snyder
R. S. Dixon
W. J. Luyten
Y. Terzian
R. L. Duncombe
Ch. Moore-Sitterly
F. B. Wood
Ch. Fehrenbach
C. E. Worley

3. The publication of information. The way in which the Group will report on the existence and preparation of data files has not yet been firmly specified. (We are primarily concerned with data files that exist on magnetic tape, but card files and other lists of data may need to be considered in some fields.) It would be impracticable, even if it were desirable, for the Group to publish detailed lists of information about individual files. Rather, the Group will endeavour to publish lists of those institutions, or individuals, that will provide detailed information about the contents and availability of files on particular topics. In some cases these institutions may act as data centres, i.e. they will hold the data files and will be prepared either to make copies available to others on a routine basis, or to search the data-base to answer particular enquiries. In other cases, the institutions may merely act as reference centres, i.e. they may publish detailed lists showing where data files are held or may refer enquiries to an appropriate centre or individual. Some Commissions have already made arrangements for the collection and publication of information about the existence and availability of data files in their fields of interest. It is hoped that other Commissions will consider how best they can promote similar activities that would be helpful to the astronomical community.

It is not possible to give a comprehensive list of sources of information, but it is hoped that the following short list will be of value.

The Bureau International d'Information sur les Ephémérides Astronomiques, at Bureau des Longitudes, Paris, distributes printed cards giving information about the availability of solar system ephemerides and positional star catalogues. (See Trans. IAU, 14B (1970), 79 and 84-5, 1971, for reports of the discussions in Commission 4 concerning the setting up of this reference centre.)

The Centre de Données Stellaires, at Observatoire de Strasbourg, has published several information bulletins. In addition to giving details of the Centre's activities in collecting physical and positional data on stars, these bulletins also contain short articles and reports on other relevant topics 
(e.g. lists of errors in catalogues, identification of cluster stars). In view of the wide coverage in content and extensive distribution of these bulletins, it is unnecessary for details of individual projects to be listed here.

The Planetary Research Centre, at Lowell Observatory, acts as an international data centre. At present it is primarily concerned with the cataloguing and documentation of planetary imaging data, but is receptive to suggestions concerning other data listings that would be of service in this field. The Lunar Science Institute, Houston, is similarly placed in regard to lunar data.

The institutions that comprise the Federation of Astronomical and Geophysical Services (FAGS) are primarily concerned with the collection, analysis and publication of data of relevance to solar physics and geophysics, including time and polar motion. The system of World Data Centres originally set up in connection with the International Geophysical Year, also hold data of relevance to some fields of astronomy. The International Committee on Data for Science and Technology (CODATA) publishes information in its Newsletters and Bulletins about data compilations in physics and chemistry, and is now extending its coverage to other sciences; and it is also concerned with the techniques for handling and evaluation of data. It appears that astronomers can hope to benefit from the past experiences and current activities of these various organisations.

4. The provision of advice. Although the Chairman has received comments on various questions that have been raised since the Group was formed, it is not yet practicable for the Group to make any specific recommendations or advice on the preparation of data files and the retrieval of information from them. In some astronomical fields there has already been considerable co-operative activity, and sophisticated data centres have been in existence for many years in other more richlyendowed branches of sciences, but it is not yet clear whether it is possible to generalise from the experiences so gained. The following remarks are therefore of a provisional character, and will be subjected to examination and amendment at the first meetings of the Group at Sydney in 1973.

(a) The bulk of astronomical data in machine-readable form is currently available on $\frac{1}{2}$-inch 9-track magnetic tape written to IBM 360 and ICL System 4 standards. The use of 7-track tape to IBM 7090 and ICL 1900 standards is becoming less common, and other types of tape are only in very limited use. In some cases the incompatibilities of the hardware are so great that data must be supplied on punched-cards or paper-tape, but even with these media there are often similar problems. The software problems of code conversion are less troublesome, provided that the data files are written in character (alphanumeric) formats and not in packed binary formats.

(b) There are several other factors that inhibit the exchange of data files. Small institutions, and individuals, are deterred from offering to make their data files available since the work involved in making the extra copies would be significant, or since the costs of materials and computer time would be high. Large institutions can usually fit in the copying work more easily, but are often subject to definite charging policies or other restrictions. Except where there is a two-way exchange of data, or where a data centre has been specially set up to receive and supply data freely, it is becoming accepted that the recipient must expect to pay for the costs of materials, copying time and postage - the total is likely to be much less than the costs of collecting, punching and checking the data again. The alternative system, whereby the requestor is expected to supply multiple quantities of blank tape or cards is not generally favoured as it often involves delay and significant packing and postage costs that do not benefit either party to the transaction.

(c) The main factor that appears to influence the success, or otherwise, of a data centre is not its size, nor its hardware facilities, nor its location, but rather whether the persons responsible for maintaining the data files take an active interest in the generation and analysis of the data. When such an interest exists, any inadequacies in accuracy or content of the data will become apparent, and a continual improvement in the data-base and in the retrieval facilities may be expected. The scope of a data centre should not be extended beyond the interests of the persons who are available at the centre, and these persons require both astronomical and data handling experience and knowledge.

(d) Although there have been some experiments in the on-line interrogation of astronomical data files, it is doubtful whether it is yet feasible to attempt to establish a comprehensive data-base that 
encompasses the widely varying attributes of astronomical objects. Progress towards this objective will depend on the successful solution of the problems of identification and classification, so that data from diverse catalogues may be amalgamated. The choice of the form of the data-base and the design of the interrogation system will require extensive consultations with astronomers working in many different fields and countries, and testing on restricted data sets.

(e) The rate of acquisition of observational data is increasing rapidly and so there will be a growing need for the preparation of tabulations of 'critical data'. Up to now the onus for this has been largely left to individuals, but in some fields (e.g. positional astronomy) there already exist both formal and informal arrangements to ensure that data of high quality are available for general use. The extension of co-operation in this type of activity must be the responsibility of the individual Commissions, but the Group could encourage such activity and could endeavour to pass on general advice from those who have had much experience of this type of work.

(f) The information so far received suggests that there are some requirements for additional reference centres (similar to that for astronomical ephemerides) in the newer fields of astronomy, and possibly even some co-operative arrangements for data collection. A fine example of the value of early action is provided by the adoption of standard formats for lunar laser-ranging data, thus providing new groups with guidance on the extent and precision to which observational data should be recorded.

5. Conclusions. It is hoped that this report on the preliminary activities of the IAU Working Group on Numerical Data will be useful in that it indicates:

(a) those persons and institutions that are willing to provide information and advice about astronomical data; and

(b) the role that the Group can play in the activities of the Union.

Although the Chairman and members of the Group will be glad to receive information about all data activities in the Union, it must be emphasised that the responsibility for encouraging cooperation and disseminating information in specialised fields rests with the Commissions concerned.

G. A. WILKINS

Chairman of the Working Group 